\title{
Clustered telomeres in phase-separated nuclear condensates engage mitotic DNA synthesis through BLM and RAD52
}

\author{
Jaewon Min, Woodring E. Wright, and Jerry W. Shay \\ Department of Cell Biology, University of Texas Southwestern Medical Center, Dallas, Texas, 75390, USA
}

\begin{abstract}
Alternative lengthening of telomeres (ALT) is a telomerase-independent telomere maintenance mechanism that occurs in a subset of cancers. One of the hallmarks of ALT cancer is the excessively clustered telomeres in promyelocytic leukemia (PML) bodies, represented as large bright telomere foci. Here, we present a model system that generates telomere clustering in nuclear polySUMO (small ubiquitin-like modification)/polySIM (SUMO-interacting motif) condensates, analogous to PML bodies, and thus artificially engineered ALT-associated PML body (APB)-like condensates in vivo. We observed that the ALT-like phenotypes (i.e., a small fraction of heterogeneous telomere lengths and formation of $\mathrm{C}$ circles) are rapidly induced by introducing the APB-like condensates together with BLM through its helicase domain, accompanied by ssDNA generation and RPA accumulation at telomeres. Moreover, these events lead to mitotic DNA synthesis (MiDAS) at telomeres mediated by RAD52 through its highly conserved N-terminal domain. We propose that the clustering of large amounts of telomeres in human cancers promotes ALT that is mediated by MiDAS, analogous to Saccharomyces cerevisiae type II ALT survivors.
\end{abstract}

[Keywords: ALT; MiDAS; phase separation; biomolecular condensates; break-induced replication]

Supplemental material is available for this article.

Received January 29, 2019; revised version accepted April 24, 2019.

Telomeres are composed of TTAGGG repeats at the ends of chromosomes. Cell divisions are accompanied by telomere length shortening; therefore, cancer cells almost universally acquire a telomere maintenance mechanism (TMM) during neoplastic transformation. Most cancers are of epithelial origin (carcinomas) and reactivate telomerase activity (Kim et al. 1994). However, cancers of mesenchymal origin, such as sarcomas and soft tissue tumors, frequently acquire a telomerase-independent TMM, which has been termed alternative lengthening of telomeres (ALT) (Bryan et al. 1997). ALT is a recombinationmediated telomere elongation process, but the underlying mechanism by which the ALT pathway is initially engaged and the molecular mechanisms underlying ALT in human cancer are still undetermined.

One of the hallmarks of ALT-positive cancer specimens is large bright telomere signals revealed by telomere fluorescence in situ hybridization (FISH) (Heaphy et al. 2011a). These bright telomere signals are clustered in promyelocytic leukemia (PML) bodies (Heaphy et al. 2011b), known as ALT-associated PML bodies (APBs). APBs contain not only telomeric DNA but also many proteins involved in DNA replication and repair processes (Yeager et al. 1999). Also, the long noncoding RNA (lncRNA) telomeric

Corresponding author: jerry.shay@utsouthwestern.edu, jaewon.min@ utsouthwestern.edu

Article published online ahead of print. Article and publication date are online at http://www.genesdev.org/cgi/doi/10.1101/gad.324905.119. repeat-containing RNA (TERRA) is also localized in APBs (Arora et al. 2014). PML bodies are one of the nuclear membrane-less organelles that are formed by liquidliquid phase separation (LLPS) and are organized by multivalent interactions between small ubiquitin-like modification (SUMO) sites and SUMO-interacting motifs (SIMs) in PML and other associated proteins (Banani et al. 2016). The relative stoichiometry of the scaffold (SUMO and SIM ratio) determines the recruitment of client proteins containing SUMOylation sites and SIMs (Ditlev et al. 2018). LLPS drives the formation of membrane-less compartments (known as biomolecular condensates) through liquid-liquid demixing from the surrounding nucleoplasm or cytoplasm and is involved in the assembly of nuclear bodies, such as PML bodies and Cajal bodies (Banani et al. 2017; Wheeler and Hyman 2018). In addition to the role of LLPS in normal cellular function, aberrant LLPS is involved in the pathogenesis of diseases, such as aggregation-related neurodegenerative disease and cancer (Aguzzi and Altmeyer 2016; Bouchard et al. 2018).

PML bodies are disassembled when cells enter mitosis (Bernardi and Pandolfi 2007; Chung et al. 2012). However,

(C) 2019 Min et al. This article is distributed exclusively by Cold Spring Harbor Laboratory Press for the first six months after the full-issue publication date (see http://genesdev.cshlp.org/site/misc/terms.xhtml). After six months, it is available under a Creative Commons License (Attribution-NonCommercial 4.0 International), as described at http://creativecommons.org/licenses/by-nc/4.0/. 
APBs are not disassembled until later in mitosis, possibly due to their hyper-SUMOylated status. Thus, they appear as APB-like foci in metaphase spreads in a subset of ALT cancer cell lines (Cesare et al. 2009). Recently, it has been shown that telomeres can be elongated during mitosis in these APB-like foci, known as mitotic DNA synthesis (MiDAS) at telomeres (Min et al. 2017b; Özer et al. 2018). However, the molecular mechanism for how telomeres cluster in PML bodies and are processed and participate in the ALT pathway are not known.

Two ALT mechanisms have been identified in the yeast Saccharomyces cerevisiae and are termed type I and type II ALT (Lundblad and Szostak 1989; Lundblad and Blackburn 1993). Type I ALT in yeast is mediated by Rad51dependent recombination, whereas type II ALT in yeast is mediated by the Rad51-independent break-induced replication process (Teng and Zakian 1999; Teng et al. 2000; Chen et al. 2001; Lydeard et al. 2007). Based on recent studies, it is now believed that these two distinct ALT mechanisms in yeast are also conserved in human ALT cancers (Verma and Greenberg 2016; Sobinoff and Pickett 2017). The type I ALT-like mechanism in human cancer is initiated by RAD51-dependent recombination and elongated by the BLM-TOP3A-RMI (BTR) dissolvase complex during S/G2 phases (Cho et al. 2014; Ramamoorthy and Smith 2015; Min et al. 2017a; Sobinoff et al. 2017). In contrast, the type II ALT-like mechanism in human cancer is mediated by a RAD51-independent pathway during G2/M phases (Henson et al. 2009; Muntoni et al. 2009; Nabetani and Ishikawa 2009; Oganesian and Karlseder 2011; O'Sullivan et al. 2014; Dilley et al. 2016; Root et al. 2016; Verma et al. 2019; Zhang et al. 2019), typically observed in APBlike foci in metaphase spreads (Min et al. 2017b).

Here, we present a biophysical model system that can reconstitute PML bodies from minimal components and generate telomere-clustered nuclear condensates and thus artificially engineered APB-like condensates in vivo. We found that the ALT-like phenotypes (i.e., a small fraction of heterogeneous telomere lengths and formation of $C$ circles) can be triggered rapidly by the reconstitution of APB-like condensates in the presence of BLM overexpression. Persistent telomere clustering in nuclear condensates leads to MiDAS at APB-like foci in metaphase through RAD52. We provide evidence that the clustering of telomeres promotes the ALT pathway mediated by mitotic telomere synthesis.

\section{Results}

Induction of telomere clustering in nuclear polySUMO/polySIM condensates can mimic the APBs in ALT cancer cells

To test whether the clustering of large amounts of telomeres in PML bodies per se is sufficient to engage the ALT pathway, we decided to use the recently developed multivalent scaffold proteins that consist of 10 or six repeats of human SUMO3 (polySUMO) and six or 10 repeats of the SIM from PIASx (polySIM) (Banani et al. 2016). The polySUMO/polySIM scaffolds can form biomolecular condensates through LLPS and functionally mimic the PML bodies in vivo (Fig. 1A). SUMO-abundant scaffold; (SUMO)10-(SIM)6 selectively recruits SIM-containing client proteins, whereas SIM-abundant scaffold;(SUMO)6(SIM)10 recruits SUMOylated client proteins (Ditlev et al. 2018). We engineered the original scaffold protein to make it (1) form the condensates in the nucleus by adding nuclear localization signals (NLS) and (2) target the telomeres to these scaffolds by adding the RAP1 C terminus (RCT) domain, which directly binds to TRF2 proteins with strong affinity (Fig. 1B; Li et al. 2000; Chen et al. 2011). We transfected plasmid DNAs containing cytomegalovirus (CMV) promoter-driven scaffold proteins in 293FT simian virus (SV40) T-antigen transformed

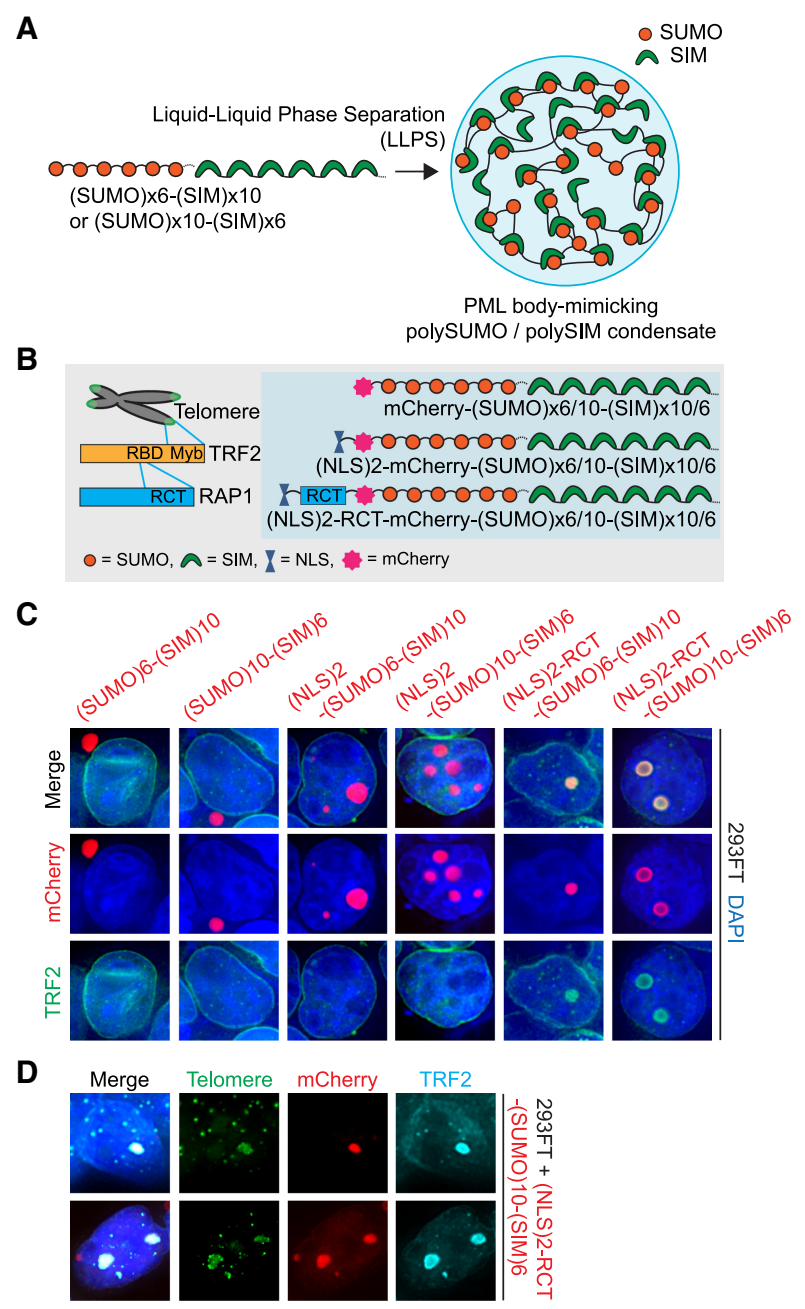

Figure 1. Engineering poly(SUMO)/poly(SIM) scaffolds to induce telomere clustering in the nucleus. $(A)$ Illustration of poly (SUMO)/poly(SIM) scaffolds [(SUMO)6-(SIM)10 or (SUMO)6(SIM)10] mimicking PML bodies in vivo. (B) Strategy for the generation of telomeres targeting nuclear condensates using NLS and $\mathrm{RCT}$ that bind to the RAP1-binding domain in TRF2. (C) Representative images showing the telomere localization of engineered poly(SUMO)/poly(SIM) scaffolds. (D) Representative images showing a telomere clustering event induced by telomere clustering scaffolds. 
human embryonic kidney cells that are telomerasepositive. The original scaffold proteins only formed the condensates in the cytoplasm due to its big size [Fig. 1C, top panel, mCherry-(SUMO)10/6-(SIM)6/10]. Adding two NLSs derived from c-Myc and SV40 to the original scaffold proteins allowed them to form condensates in the nucleus [Fig. 1C, middle panel, (NLS)2-mCherry-(SUMO)10/6(SIM)6/10]. However, adding the NLSs was not sufficient to induce telomere clustering. We additionally tagged the RCT domain to target telomeres to the condensates [Fig. 1C, bottom panel, (NLS)2-RCT-mCherry-(SUMO) 10/6-(SIM)6/10]. TRF2, a shelterin protein, colocalized mostly with the condensates. By using the telomereFISH assay, we further confirmed that telomeres colocalized mostly with the telomere clustering scaffolds (Fig. 1D). Thus, these scaffolds enabled the generation of telomere clustering in nuclear condensates (referred to as telomere clustering scaffolds), mimicking APBs and large bright telomere foci that occur in ALT cancer cells.

Next, we checked whether the polySUMO/polySIM nuclear condensate-induced telomere clustering events were sufficient to trigger other hallmarks of the ALT pathway. We transfected the plasmids expressing various scaffold proteins in 293FT cells and analyzed them $72 \mathrm{~h}$ after transfection. Contrary to our expectation, introducing telomere clustering scaffolds did not lead to any changes in telomere phenotypes, such as telomere length alterations or extrachromosomal telomere repeat (ECTR) generation (Supplemental Fig. S1; Cesare and Reddel 2010). We conclude that telomere clustering events induced by polySUMO/polySIM condensates are not sufficient to trigger the ALT pathway.
Telomere clustering induces the ALT-like phenotypes in the presence of BLM overexpression

We hypothesized that additional factors were required to trigger the ALT pathway in addition to the telomere clustering scaffold. First, we tested whether the overexpression of BLM can trigger the ALT pathway in the presence of the telomere clustering scaffold (Fig. 2A). BLM is known as a major component of the ALT pathway as well as in PML bodies (Yankiwski et al. 2000; Stavropoulos et al. 2002). The telomere clustering scaffolds were cotransfected with a BLM cDNA plasmid into 293FT cells and analyzed for ALT phenotypes, including telomere length alterations and ECTRs. We harvested the transfected cells $72 \mathrm{~h}$ after transfection and predicted that if the ALT pathway was engaged, we would observe heterogeneous telomere length using the terminal restriction fragment (TRF) analysis, and the cells would become positive using the $\Phi 29$ polymerase reaction for determining the existence of ECTRs (as detected by the C-circle assay) (Fig. 2A; Henson et al. 2009).

We found that the overexpression of the BLM helicase induced ALT-like phenotypes in the presence of telomere clustering associated with SUMO-abundant scaffolds [(SUMO)10-(SIM)6]. Co-overexpression of BLM helicase with SUMO-abundant scaffolds rapidly induced heterogeneous telomere length and complex telomere structures as observed in TRF gels, with telomeres of various sizes down to $<0.8 \mathrm{~kb}$ (a small fraction), and unmigrated telomere DNA stuck in the gel well (potentially indicating branched DNA; e.g., DNA replication fork or recombination intermediates) (Fig. 2B, top) also became C-circle-
A

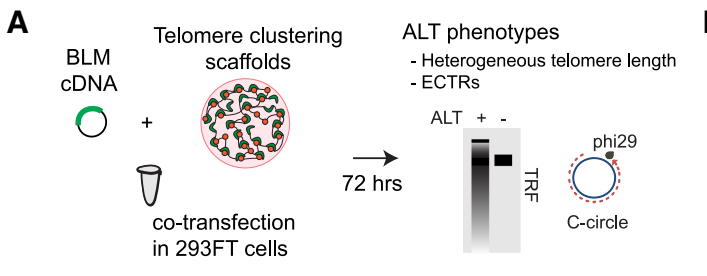

C

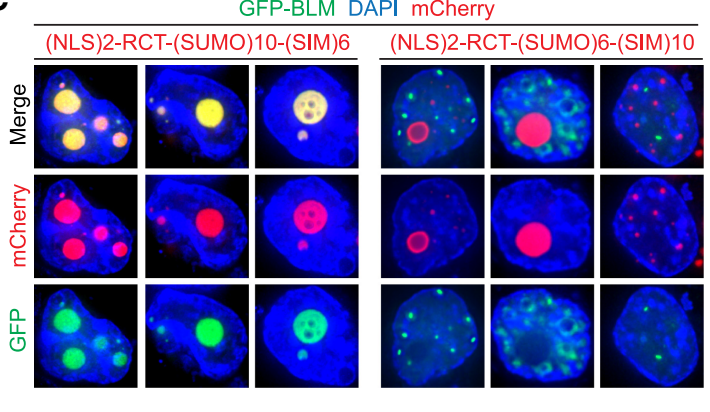

D

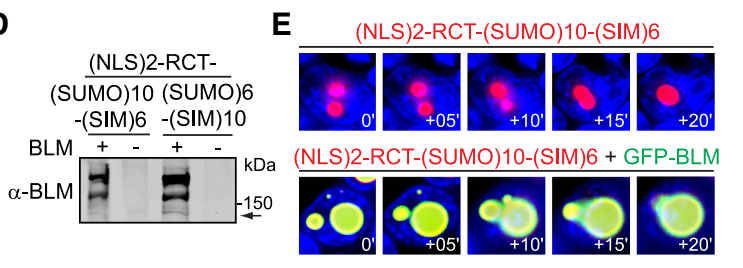

B

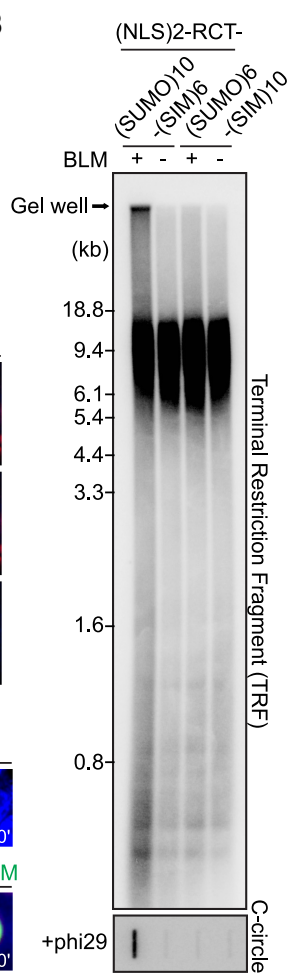

Figure 2. Induction of ALT-like phenotypes by cooverexpression of telomere clustering scaffolds with BLM helicase. (A) Illustration of experimental scheme to test whether BLM helicase can trigger the ALT pathway with the telomere clustering scaffold. (B) The TRF analysis (top) and the C-circle assay (bottom) of 293FT telomerase-positive cells after overexpression of telomere clustering scaffolds and BLM helicase (or empty vector). (C) Representative images showing the localization of telomere clustering scaffolds and GFP-BLM in 293FT cells. (D) Immunoblot of 293FT cells overexpressing GFP-BLM and telomere clustering scaffolds. The arrow indicates endogenous BLM. (E) Live-cell images of 293FT cells after overexpression of telomere clustering scaffolds and BLM. 
positive (Fig. 2B, bottom). BLM helicase localized at SUMO-abundant scaffolds, not SIM-abundant scaffolds, suggesting the possibility that the recruitment of BLM helicase is controlled by the stoichiometry of scaffold proteins (Fig. 2C). We checked that overexpression of the telomere clustering scaffolds did not affect the stability of BLM helicase protein (Fig. 2D). We also confirmed that overexpression of BLM helicase alone or with RCT-lacking SUMO-/SIM-abundant scaffolds did not induce the ALT-like phenotypes (Supplemental Fig. S2A,B).

To investigate the kinetics on the phenotypes observed, we analyzed the ALT-like characteristics at multiple time points $(0,24,48$, and $72 \mathrm{~h}$ after transfection). The complex telomere structure (unmigrated telomere DNAs stuck in the gel well) was very rapidly induced $24 \mathrm{~h}$ after transfection, whereas heterogeneous telomere lengths and C circles were gradually increased until $72 \mathrm{~h}$ (Supplemental Fig. S3). Saos2, an authentic ALT cell line, has a greater fraction of heterogeneous telomeres and more $\mathrm{C}$ circles than our model system (Supplemental Figs. S2, S3). To eliminate the possible effect of telomerase actions in the generation of these ALT-like phenotypes, we also tested TERC knockout 293FT cells, which have no telomerase activity (Min et al. 2017a). However, these cells also underwent G2/M arrest when we overexpressed telomere clustering scaffolds and thus did not exhibit the continuous proliferation in the absence of telomerase that is a hallmark of ALT. We found that the ALT-like phenotypes were also very rapidly induced in low population doublings (PDs) of TERC knockout cells (40 PDs, telomere length $<15 \mathrm{~kb}$ ), whereas ALT-like phenotypes were not induced in high PDs of TERC knockout cells (150 PDs, telomere length $<9 \mathrm{~kb}$ ) (Supplemental Fig. S4A,B). Consistent with these observations, we were not able to detect telomere clustering events induced by telomere clustering scaffolds in high PD cells (Supplemental Fig. S4C), suggesting that certain amounts of telomere lengths $(>10 \mathrm{~kb})$ are required for the induction of telomere clustering events and ALT-like phenotypes induced by telomere clustering scaffolds.

PML body formation is thought to proceed via nucleation and growth of liquid-like droplets, especially APBs that are nucleated at telomeres and undergo fusion events (Brouwer et al. 2009; Chung et al. 2011; Erdel and Rippe 2018). We found that the telomere clustering scaffolds also possessed the feature of a liquid-like droplet, as displayed by rapid fusion events among condensates (Fig. 2E). However, cells expressing telomere clustering scaffolds showed cell cycle arrest during mitosis (Supplemental Fig. S5), possibly due to the C-terminal diglycine motif in SUMO proteins in polySUMO/polySIM constructs that are mutated so that the condensates are retained persistently and do not dissolve during mitosis (Banani et al. 2016).

\section{SUMOylations/SIMs in BLM protein determine its} recruitment to the telomere clustering scaffolds

The BLM helicase protein has three major SUMOylation sites (K317, K331, and K344) and two SIMs (QIDL [amino acids 216-219] and VICI [235-238]) that are required for the localization of BLM in PML bodies (Supplemental Fig. S6A; Eladad et al. 2005; Zhu et al. 2008; Hendriks et al. 2017). However, it is unknown how these SUMOylation sites and SIMs in BLM protein are collaborating to control its localization to PML bodies. We tested whether the status of SUMOylation and SIM in BLM protein can determine the recruitment of BLM to the scaffolds. We constructed SUMO-defective (triple lysine [K] to arginine [R] [TKR]: K317R, K331R, and K344R), SIM-defective (sim: QIDL to QADA, VICI to AACI), and both SUMO- and SIM-defective (double mutant [TKRsim]) mutants. BLM sim or TKRsim mutants were not recruited to the SUMO-abundant scaffold, whereas the BLM TKR mutant was comparable with wild-type (WT) (Supplemental Fig. S6B). Consequently, overexpression of BLM sim or TKRsim mutants did not induce ALT-like phenotypes (Supplemental Fig. S6C). Since BLM may not be SUMOylated in 293FT cells, we constructed the three repeats of SUMO1 and BLM helicase fusion protein (Supplemental Fig. S7A). (SUMO1)3-BLM proteins were recruited to two different telomere clustering scaffolds, and the ALTlike phenotype was induced in the presence of both SUMO-abundant and SIM-abundant scaffolds (Supplemental Fig. S7B,C). We interpret these results to suggest that the recruitment of BLM helicase is determined by the ratio of SUMO and SIM in telomere clustering scaffolds, as the recruitment of the client protein is controlled by the stoichiometry of scaffold proteins in cellular bodies (Supplemental Fig. S7D).

The helicase activity of BLM involved in long-range resection is required for the induction of the ALT-like phenotypes

BLM has two distinct functions. First, BLM forms a complex with the TOP3A and RMI (BTR) "dissolvasome" required for the dissolution processes during bubble migration (Bizard and Hickson 2014). Second, BLM cooperates with DNA2 by forming a helicase/nuclease complex involved in long-range resection processes $\left(5^{\prime}\right.$-to- $3^{\prime}$ resection) at the end of double-stranded breaks (Nimonkar et al. 2011). This led us to investigate the domain of BLM protein involved in the induction of the ALT-like phenotypes. We constructed the BTR complex-defective (btr [K3A]: K38A/K39A/K40A) mutant and the helicasedead (K695A) mutant (Supplemental Fig. S8A; Bugreev et al. 2007; Wang et al. 2013; Blackford et al. 2015). The BLM K695A mutant did not induce the ALT-like phenotype, whereas the BLM btr mutant was comparable with WT and did induce the ALT-like phenotype (Supplemental Fig. S8B). The long-range resection processes mediated by BLM-DNA2 may generate ssDNA accumulation and ultimately lead to RPA protein accumulation at the resected ssDNA (Nimonkar et al. 2011; Bhat and Cortez 2018). We thus checked whether RPA and phospho-RPA proteins localize at the BLM-recruited clustered telomeres. BLM WT and btr mutant-expressing cells showed more RPA and phospho-RPA accumulation compared with BLM K695A and mock control (empty vector) 


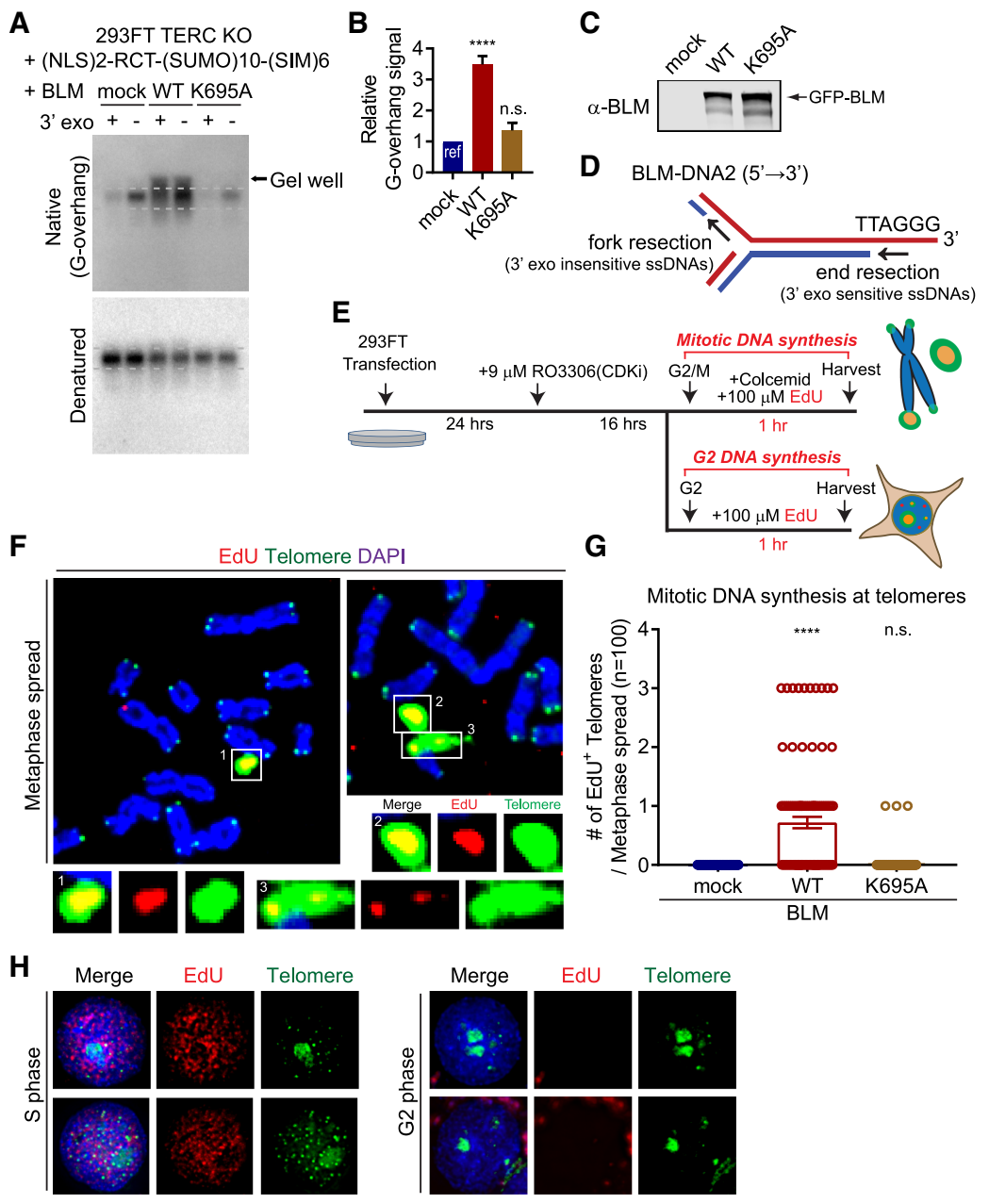

Figure 3. BLM helicase activity is required for the induction of ALT-like phenotypes mediated by $\mathrm{Mi}$ DAS. (A) In-gel hybridization analysis of 293FT TERC knockout cells with overexpression of telomere clustering scaffolds and BLM WT or K695A (helicase-dead) mutant or empty vector. (B) Quantification of G-rich single-stranded telomere intensity. Relative ratio of native signal to denatured signal was calculated. Mean \pm SEM. $n=3$. (C) Immunoblot of 293FT TERC knockout cells represented in $A$. (D) Model for the generation of G-rich single-stranded telomere by long-range resection processes mediated by BLM-DNA2. (E) Experimental scheme for G2-phase/mitotic telomere synthesis analysis. EdU (5-ethynyl-2-deoxyuridine) represents newly synthesized DNA during mitosis (top; metaphase chromosome) or G2 phase (bottom; interphase cell). $(F, G)$ Telomeric MiDAS analysis of 293FT TERC knockout cells with overexpression of telomere clustering scaffolds and BLM WT or K695A (helicase-dead) mutant or empty vector. $(F)$ Representative images showing MiDAS at telomeres on metaphase spreads. $(G)$ Quantification of telomeric MiDAS and the number of EdU-positive telomeres per metaphase spread. Means \pm SEM. $(H)$ Representative images showing S phase (left) or G2 phase (right) of 293FT TERC knockout cells with overexpression of telomere clustering scaffolds and BLM WT. $\left(^{* * * *}\right) P<0.0001$; (n.s.) nonsignificant, unpaired Student's $t$-test.
(Supplemental Fig. S8C-E). Next, we directly measured the amount of single-stranded telomere DNA using the in-gel hybridization assay. To eliminate the amounts of overhangs elongated by telomerase, we used 293FT TERC knockout cells. Overexpression of BLM WT generated more G-rich single-stranded telomere DNA compared with mock control and the K695A mutant (Fig. $3 \mathrm{~A}-\mathrm{C})$. Moreover, the DNA was partially sensitive to the exonuclease I reaction (Fig. 3A, $3^{\prime}$ exo lanes in native conditions), indicating that those ssDNAs contain a substantial portion of internal gaps (e.g., potentially generated by DNA replication fork resection; exonuclease Iinsensitive ssDNAs) as well as $3^{\prime}$ ssDNAs (e.g., generated by end resection; exonuclease I-sensitive ssDNAs) (Fig. 3D). Indeed, overexpression of BLM WT in the absence of telomere clustering scaffolds did not induce any changes in G-rich single-stranded telomere DNA (Supplemental Fig S9A-C). We interpret these data to support the idea that single-stranded telomere DNA generation processes are involved in the induction of the ALT phenotypes (Nabetani and Ishikawa 2009; Oganesian and Karlseder 2011; O'Sullivan et al. 2014; Flynn et al. 2015; Doksani and de Lange 2016; Mao et al. 2016; Min et al. 2017a).

\begin{abstract}
ALT-like phenotypes induced by telomere clustering and BLM overexpression are associated with MiDAS at telomeres
\end{abstract}

The helicase activity of BLM may be required for the association of ECTRs in APBs with chromosomal telomeres in response to DNA replication stresses (Komosa et al. 2015; Root et al. 2016). Moreover, overexpression of telomere clustering scaffolds induces persistent telomere clustering, leading to G2 and $M$ arrest (Supplemental Fig. S5). This led us to propose that the single-stranded telomere DNAs generated by BLM-DNA2-mediated long-range resection processes can be replicated and repaired during mitosis through MiDAS.

We tested whether overexpression of telomere clustering scaffold with BLM protein leads to MiDAS at telomeres. The CDK inhibitor (treated at $24 \mathrm{~h}$ after transfection) was used to synchronize cells (5-ethynyl-2-deoxyuridine) before cell cycle progression was perturbed by overexpression of the telomere clustering scaffold and BLM protein (Fig. 3E). EdU (5-ethynyl-2-deoxyuridine) was pulsed for $1 \mathrm{~h}$ during late $\mathrm{G} 2 / \mathrm{M}$ phase to identify newly synthesized DNA during mitosis (as determined by analyzing metaphase chromosomes) or G2 phase (as 
determined by analyzing interphase cells). We found that co-overexpression of the telomere clustering scaffold with BLM protein led to mitotic telomere synthesis (Fig. 3F). Moreover, there was an excessive amount of EdU labeling in large and bright telomere signals (Fig. 3F, cropped squares), comparable with APB-like foci frequently observed in a subset of ALT cancer cells that are associated with chromosome ends or exist as extrachromosomal telomeric DNA (Cesare et al. 2009; Min et al. 2017b). BLM WT induces a significant number of telomeric MiDAS, whereas BLM K695A mutant or mock control did not (Fig. 3G). However, we were not able to detect any EdU incorporation in telomere clustering scaffolds during $\mathrm{S}$ or $\mathrm{G} 2$ phases (Fig. $3 \mathrm{H}$ ), indicating that telomeric DNA was potentially underreplicated during $S$ phase and not processed during G2 phase in telomere clustering scaffolds. We conclude that long-range resection processes by BLM helicase is required for triggering telomeric MiDAS in the presence of telomere clustering scaffolds.

\section{$B L M$ helicase is involved in the induction of the ALT pathway during G2 and $M$ phases in ALT cancer cells}

To investigate whether the BLM helicase is involved in the induction of the ALT pathway mediated by MiDAS in human ALT cancer cells, we used Saos2 cells, which are an ALT cancer cell line derived from an osteosarcoma patient. Saos 2 cells display large telomere foci as determined by telomere-FISH (Cox et al. 2016), as was shown in ALT cancer tissue sections (Heaphy et al. 2011a,b). Moreover, Saos2 cells harbor severe DNA replication stresses at telomeres and deficiency in a G2/M checkpoint that leads to persistent DNA damage responses and MiDAS at telomeres (Cesare et al. 2009; Lovejoy et al. 2012; Min et al. 2017b). Thus, Saos2 cells are suitable for further examining a human ALT model. We introduced lentiviral CMV promoter-driven BLM WT and various mutants, including sim, TKR, TKRsim, and K695A, into Saos2 cells (Supplemental Fig. S10A). Consistent with previous reports, BLM protein localized at PML bodies and telomeres in ALT cells (Stavropoulos et al. 2002; Déjardin and Kingston 2009; Pan et al. 2017; Sobinoff et al. 2017). While BLM sim and TKRsim mutants formed many big foci, none of them localized at telomeres and PML bodies (Supplemental Fig. S10B,C). In contrast, BLM TKR proteins localized at PML bodies and telomeres, although a portion of BLM TKR proteins formed PML-independent foci (Supplemental Fig. S10B,C, TKR), indicating that SIMs in BLM protein are the major factor recruiting BLM to APBs. Intriguingly, BLM K695A mutants were recruited to PML bodies and telomeres; however, most were not clustered (Supplemental Fig. S10B,C, K695A). We next checked whether the telomere clustering, APB formation, and C-circle generation in Saos 2 cells are altered by BLM mutant overexpression. Overexpression of BLM WT increased telomere clustering, APB formation, and C-circle levels, whereas BLM sim or TKRsim overexpression decreased them, indicating that the BLM sim and TKRsim are dominant-negative mutants (Fig. 4A-D, WT, sim, and TKRsim). Interestingly, BLM
TKR overexpression increased telomere clustering events and C-circle levels, whereas it did not lead to any significant changes in APB formation (Fig. 4A-D, TKR), suggesting the possibility that $\mathrm{C}$-circle generation in BLM TKR is independent of APB formation, since BLM TKR proteins can form PML-independent foci (Supplemental Fig. S10B,C, TKR; Eladad et al. 2005). BLM K695A overexpression decreased telomere clustering but caused no significant changes in C-circle levels and APB formation. However, the size of APB foci was smaller than controls, indicating that the BLM K695A mutant exhibits a dominant-negative effect on telomere clustering (Fig. 4A-D, K695A). Finally, we measured G2 phase and mitotic telomere synthesis in BLM WT or various mutants overexpressed in Saos 2 cells (Fig. 4F,H). Overexpression of BLM WT or the TKR mutant led to increases in G2 phase and mitotic telomere synthesis, whereas overexpression of BLM sim, TKRsim, or K695A mutants led to a decrease in G2 phase and mitotic telomere synthesis (Fig. 4G,I). We interpret these results to indicate that BLM proteins are recruited to APBs through SIMs, and the helicase activity of BLM is required for telomere clustering events, ultimately leading to the induction of the ALT pathway.

It has been reported that BLM protein frequently localizes at telomeres in ALT cells, potentially by ALT cellspecific interaction with TRF2 (Stavropoulos et al. 2002). Moreover, TRF2 protein is SUMOylated by the SMC5/6 complex in ALT cells, and its SUMOylation is required for APB formation (Potts and Yu 2007). We next tested whether the status of SUMOylation of TRF2 affects telomere clustering and telomeric MiDAS. We constructed the SUMO-defective (sextuple lysine [K] to arginine [R] [SKR]: K245R, K293R, K331R, K327R, K333R, and K410R) mutant (TRF2 SKR) and the three repeats of SUMO1 and SUMO-defective mutant fusion protein [(SUMO)3-TRF2 SKR] (Fig. 5A). We introduced lentiviral CMV promoterdriven TRF2 WT, SKR mutant, and (SUMO)3-TRF2 SKR proteins into endogenous TRF2-depleted Saos2 cells (Fig. $5 \mathrm{~B}, \mathrm{C})$. Saos 2 cells expressing the TRF2 SKR mutant exhibited defects in telomere clustering and APB formation, whereas cells expressing the SUMO1-tagged TRF2 SKR mutant displayed enhanced telomere clustering and APB formation (Fig. 5D,E). Furthermore, telomere synthesis during G2 and M phases decreased in the TRF2 SKR mutant but increased in the (SUMO1)3-TRF2 SKR mutant (Fig. 5F-I). These results support the idea that SUMOylation events at telomeres are required for the telomere clustering and induction of the ALT pathway (Potts and Yu 2007; Chung et al. 2011; Min et al. 2017b).

\section{RAD52 participates in the induction of ALT-like phenotypes}

It has been reported that MiDAS is mediated by RAD52dependent, but RAD51-independent, break-induced replication processes (Minocherhomji et al. 2015; Bhowmick et al. 2016). RAD52 also facilitates telomeric MiDAS in both APB-like foci and chromatid ends (Min et al. 2017b; Özer et al. 2018). RAD52 consists of two distinct domains: the N-terminal domain (NTD) and the C-terminal domain 
A

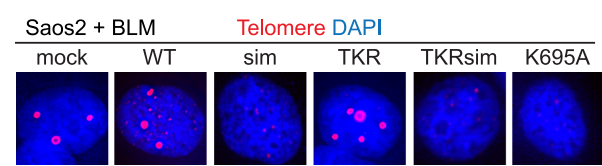

C

Saos2 + BLM PML Telomere DAPI

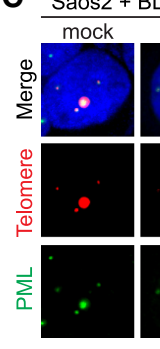

E

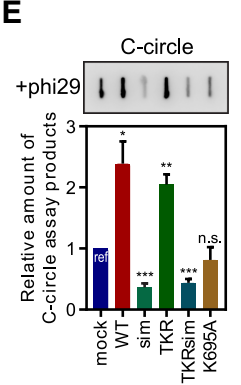

H

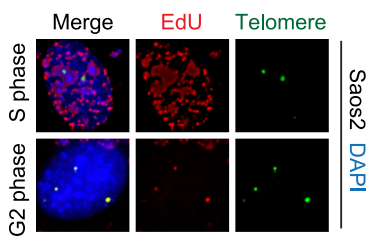

G

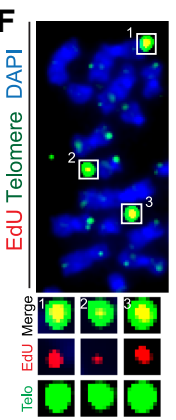

B

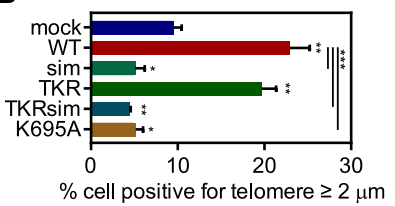

D
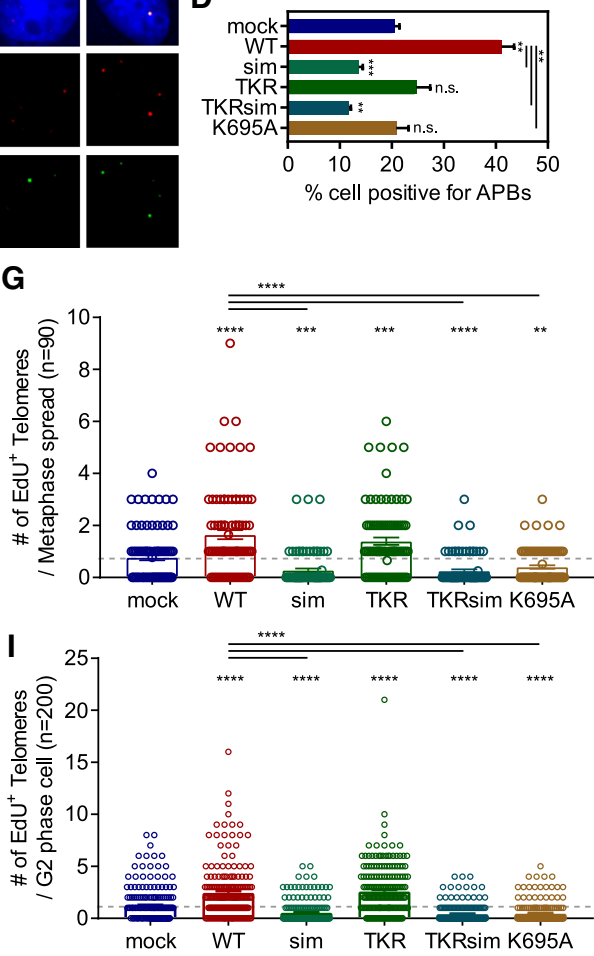

Figure 4. ALT mediated by MiDAS is facilitated by BLM helicase. $(A, B)$ Telomere clustering analysis of Saos2 cells in the presence of BLM WT or BLM mutant overexpression. (sim) SIM-defective (sim: QIDL to QADA, VICI to AACI); (TKRsim) SUMO- and SIM-defective (double mutant); (K695A) helicase-dead. (A) Representative images showing telomere clustering. (B) Quantification of telomere clustering as the percentage of cells containing $\geq 2 \mu \mathrm{M}$ clustered telomeres. Means \pm SEM. $n=4(C, D)$ APB analysis of Saos2 cells in the presence of BLM WT or BLM mutant overexpression. (C) Representative images showing the colocalization of PML and telomeres. $(D)$ Quantification of APBs as a percentage of cells containing PML bodies at telomeres. Means \pm SEM. $n=4$. $(E)$ C-circle assay for Saos2 cells in the presence of BLM WT or BLM mutant overexpression. (Top) Slot blot images for C-circle assay. (Bottom) Quantification of C-circle assay as the relative amount of C-circle assay products. Means \pm SEM. $n=3 .(F, G)$ Telomeric MiDAS analysis of Saos2 cells in the presence of BLM WT or BLM mutant overexpression. $(F)$ Representative images showing MiDAS at telomeres on metaphase spreads. $(G)$ Quantification of telomeric MiDAS as the number of EdU-positive telomeres per metaphase spread. Means \pm SEM. $(H, I)$ G2-phase telomere synthesis analysis of Saos2 cells in the presence of BLM WT or BLM mutant overexpression. $(H)$ Representative images showing telomere synthesis during S or G2 phase. (I) Quantification of G2-phase telomere synthesis as the number of EdU-positive telomeres per cell. Means \pm SEM. $\left(^{*}\right) P<0.05 ;\left(^{* *}\right) P<0.01 ;\left({ }^{* * *}\right) P<$ 0.001 ; (***) $P<0.0001$; (n.s.) nonsignificant, unpaired Student's $t$-test.
(CTD). The NTD is also known as the highly conserved region (HCR), especially since it shares $42 \%$ amino acid sequence identity between $S$. cerevisiae and humans (Hanamshet et al. 2016). The RAD52 NTD is involved in ssDNA and dsDNA binding and RAD52 multimerization, whereas the CTD consists of two parts involved in interactions with RPA and RAD51 (Fig. 6A). The RAD52 NTD protein without the CTD itself can form the multimerization and participate in various recombination processes, such as single-strand annealing and RNA-templated DNA repair (Singleton et al. 2002; Mazina et al. 2017; McDevitt et al. 2018). However, the exact domain of RAD52 by which MiDAS and break-induced replication are mediated is undetermined.

We decided to test whether RAD52 is responsible for the ALT-like phenotypes that we observed. We generated RAD52 knockout in 293FT TERC knockout cells (Fig 6B; Supplemental Fig. S11A-C). Since the RAD51-interacting region in the RAD52 CTD is involved in the regulation of RAD51 during homologous recombination, RAD52-deficient cells harbor mild defects in RAD51 focus formation in response to DNA double-stranded breaks (Lok et al. 2013; Teng et al. 2018). A RAD52 knockout clone displayed partial defects in RAD51 focus formation in response to ionizing radiation (IR) (Fig. 6C). When telomere clustering scaffolds and BLM proteins were overexpressed, ALT-like phenotypes, heterogeneous telomere length, and telomeric MiDAS were not induced in the RAD52 knockout clone, whereas they were induced in the RAD52 WT clone (Fig. 6D-F, WT and knockout). We also confirmed these observations by introducing RAD52 shRNA into 293FT cells (Supplemental Fig. S12A). Consistently, the ALT-like phenotype was not induced in RAD52-depleted cells (Supplemental Fig. S12B). Moreover, introducing the RAD52 HCR was sufficient to rescue the RAD52 deficiency, suggesting that RAD52 is involved in these processes, possibly through its NTD (Supplemental Fig. S12C,D). However, C-circle levels were not significantly changed in the RAD52-depleted conditions (Fig. 6D; Supplemental Fig. S12B,D), suggesting that the C-circle generation is not completely dependent on RAD52 status.

Interestingly, we observed that the RAD52 WT clone displayed a telomeric sequence insertion (TSI) on metaphase chromosomes when telomere clustering scaffolds and BLM proteins were overexpressed (Fig. 6G-H). These events are potentially derived from aberrant recombination between the telomeres and other genomic regions. 
A

TRF2 SUMOylation sites (K): $245,293,311,327,333,410$

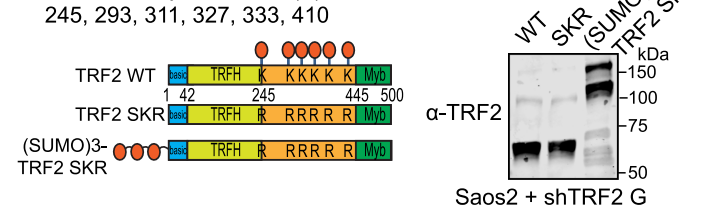

D

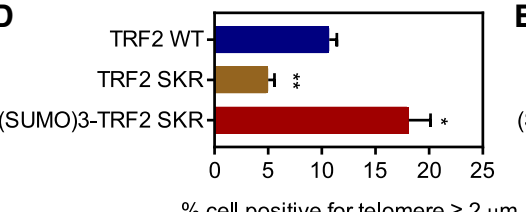

E (SUMO)3-TRF2 SKR

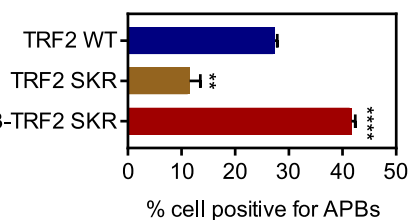

$\mathbf{F}$

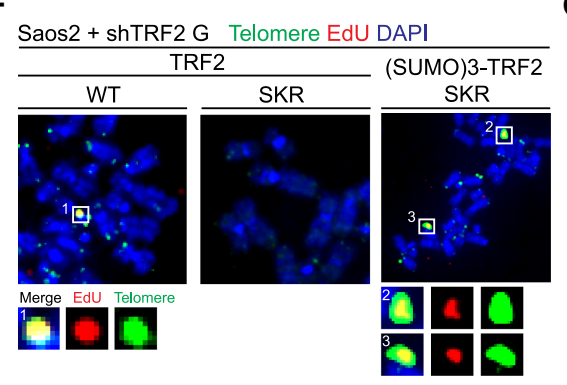

H

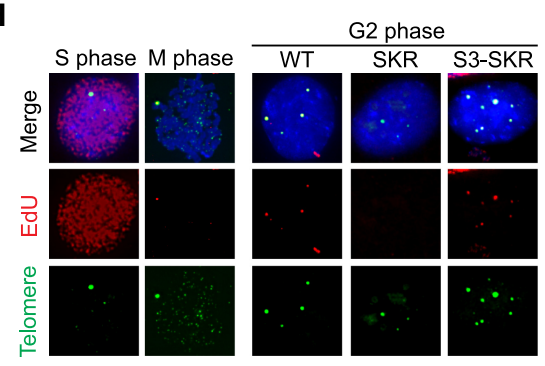

G
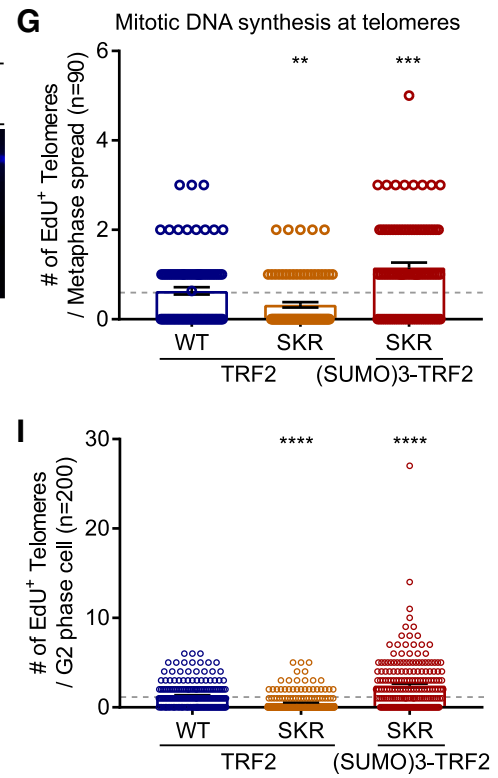

Figure 5. SUMOylation of TRF2 is required for the ALT pathway. (A) Illustration of SUMOylation sites in the TRF2 protein, SUMO-defective TRF2 mutant (TRF2 SKR), and SUMO-fused SUMOdefective TRF2 mutant [(SUMO)3-TRF2 SKR]. (B) Immunoblot of endogenous TRF2-depleted Saos 2 cells in the presence of shTRF2 G-resistant TRF2 WT or TRF2 mutant overexpression. (C) Representative images showing the telomere localization of TRF2 WT and TRF2 mutants. (D) Telomere clustering analysis of endogenous TRF2-depleted Saos2 cells in the presence of shTRF2 G-resistant TRF2 WT or TRF2 mutant overexpression. Quantification of telomere clustering as the percentage of cells containing $\geq 2 \mu \mathrm{M}$ clustered telomeres. Means \pm SEM. $n=3$. (E) APB analysis of endogenous TRF2-depleted Saos2 cells in the presence of shTRF2 G-resistant TRF2 WT or TRF2 mutant overexpression. Quantification of APBs as a percentage of cells containing PML bodies at telomeres. Means \pm SEM. $n=3$. $(F, G)$ Telomeric MiDAS analysis of endogenous TRF2-depleted Saos2 in the presence of shTRF2 G-resistant TRF2 WT or TRF2 mutant overexpression. $(F)$ Representative images showing MiDAS at telomeres on metaphase spreads. $(G)$ Quantification of telomeric MiDAS as the number of EdU-positive telomeres per metaphase spread. Means \pm SEM. $(H, I)$ G2-phase telomere synthesis analysis of endogenous TRF2-depleted Saos2 in the presence of shTRF2 G-resistant TRF2 WT or TRF2 mutant overexpression. $(H)$ Representative images showing S-phase, G2-phase, or mitotic telomere synthesis. (I) Quantification of G2-phase telomere synthesis as the number of EdU-positive telomeres per cell. Means \pm SEM. $\left({ }^{*}\right) P<0.05 ;\left({ }^{* *}\right) P$ $\left.<0.01 ;\left(^{* * *}\right) P<0.001 ;(* * *)\right) P<0.0001$, unpaired Student's $t$-test.
We interpret these results to indicate that the RAD52 is required for the induction of ALT-like phenotypes but also leads to rearrangements between telomeres and genomic regions, something observed previously in ALT cells (Marzec et al. 2015).

\section{RAD52 participates in the ALT pathway through its NTD in ALT cancer cells}

We next checked whether RAD52 is involved in the ALT pathway though the RAD52 NTD. We introduced lentivirus expressing mCherry-fused RAD52 WT or HCR-RPA (HCR and RPA-interacting region) or HCR mutants (Fig. 7A) into Saos 2 cells. Two NLSs were tagged to mCherry because mCherry-fused RAD52 mutants did not localize at the nucleus due to the increased size from mCherry fusion and the NLS deletion in their $\mathrm{C}$ termini. We found that RAD52 WT, HCR-RPA, and HCR proteins formed foci at telomeres, although a portion of RAD52 HCR proteins were spread throughout the nucleus and formed several nontelomeric foci (Fig. 7B), suggesting that the interaction with RPA may regulate the recruitment of RAD52 to telomeres in ALT cells.
We tested whether overexpression of RAD52 WT or mutants into Saos2 cells can lead to alteration of the ALT pathway. Overexpression of RAD52 WT, HCRRPA, or HCR led to increases in G2 phase and mitotic telomere synthesis (Fig. 7C-F, +RAD52 panels). As a comparison, we introduced RAD52 shRNA into Saos2 cells (Supplemental Fig. S13A), which led to a significant decrease in MiDAS at telomeres (Fig. 7C,D, shRAD52 panel), consistent with previous results done by siRNAs targeting RAD52 (Min et al. 2017b; Özer et al. 2018). However, RAD52 depletion led to only a partial decrease in telomere synthesis during G2 phase (Fig. 7E,F, shRAD52 panel). Interestingly, we found that overexpression of RAD52 WT and HCR-RPA and HCR mutants increased telomere clustering and APB formation, whereas RAD52 depletion decreased them (Supplemental Fig. S13B-E). We conclude that RAD52 is involved in the ALT pathway through the RAD52 NTD.

\section{Discussion}

We developed a model system that is similar to an ALT cancer-specific phenotype: large bright telomere foci 


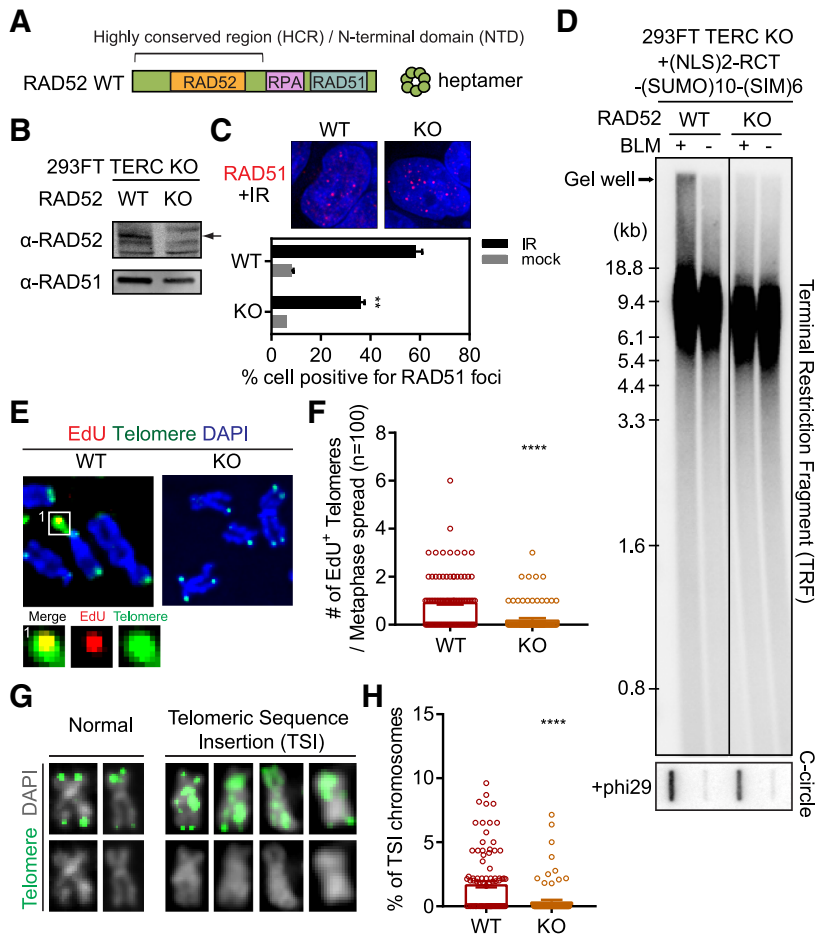

Figure 6. RAD52 is required for the induction of ALT-like phenotypes mediated by MiDAS. (A) Illustration of human RAD52 protein and its domains. The highly conserved NTD is involved in multimerization. (B) Immunoblot of RAD52 WT or knockout (KO) derived from 293FT TERC knockout by introducing CRISPR/Cas9 protein and guide RNA targeting RAD52 gene. The arrow indicates the RAD52 protein. (C) RAD51 focus formation analysis for RAD52 WT and knockout. (Top) Representative images showing RAD51 focus formation after 15-Gy of ionizing radiation (IR). (Bottom) Quantification of RAD51 focus formation as a percentage of cells containing RAD51 foci. Means \pm SEM. $n=$ 4. (D) TRF analysis (top) and the C-circle assay (bottom) of RAD52 WT and knockout after overexpressing telomere clustering scaffold and BLM (or empty vector as a negative control). (E,F) Telomeric MiDAS analysis of RAD52 WT and knockout after overexpression of telomere clustering scaffolds and BLM. (E) Representative images showing MiDAS at telomeres on metaphase spreads. $(F)$ Quantification of telomeric MiDAS as the number of EdU-positive telomeres per metaphase spread. Means \pm SEM. $(G, H)$ Telomeric sequence insertion (TSI) analysis of RAD52 WT and knockout after overexpression of telomere clustering scaffolds and BLM. $(G)$ Representative images showing TSI on metaphase spreads. $(H)$ Quantification of TSI as a percentage of TSI-positive. Means \pm SEM. (**) $P<0.01 ;(* * *) \quad P<0.0001$, unpaired Student's $t$-test.

(observed in ALT cancer tissue telomere-FISH) representing the clustering of large amounts of telomeres in PML bodies. By using polySUMO/polySIM condensates targeting telomere regions, analogous to APBs, we demonstrated that telomere clustering events are required for the engagement of the ALT pathway mediated by MiDAS. When the polySUMO/polySIM nuclear condensates are introduced into telomerase-positive cells together with BLM in the presence of endogenous RAD52, the ALT phenotype is recapitulated very rapidly, including $\mathrm{C}$ circles, heterogeneous telomere lengths, and complex telomere structures (Supplemental Fig. S3). Moreover, we identified two additional components of ALT-BLM, and RAD52that participate in these processes stepwise through their different functions. We dissected the underlying mechanisms and observed that the helicase activity of BLM protein (involved in $5^{\prime}$-to- $3^{\prime}$ resection processes) and the multimerization and DNA-binding activity of RAD52 (which has annealing activity) are participating in these processes. The key biological role of ALT is the maintenance of telomeres in the absence of telomerase. Refining our artificially engineered system to more closely reflect in vivo events, thus allowing continuous cell proliferation, will provide further insight into APB formation and the ALT pathway.

BLM protein has been implicated in the ALT pathway as well as telomere replication processes (Sfeir et al. 2009; Barefield and Karlseder 2012; Zimmermann et al. 2014; Li et al. 2018). In the present studies, we demonstrated that BLM helicase is required for the initiation of telomere clustering and telomeric MiDAS through its helicase activity by cooperating with DNA2, which is responsible for the generation of single-stranded telomeric DNAs via long-range resection processes. Consistent with our observation, it has been proposed that BLM helicase activity is required for the association of ECTRs with chromatid ends in APBs (Stavropoulos et al. 2002; Root et al. 2016). Moreover, human BLM protein can rescue the type II ALT pathway through its helicase activity in telomerasenegative sgs1 yeast, the telomerase, and the BLM homolog double mutant (Lillard-Wetherell et al. 2005). We thus conclude that BLM helicase activity is required for the initiation of the ALT pathway through the generation of single-stranded telomeric DNAs, which may be involved in annealing processes with other potential templates, such as chromosomal telomeres, ECTRs, or TERRA.

RAD52 protein has been implicated recently for its NTD novel functions through its annealing activity between ssDNA-ssDNA, ssDNA-dsDNA, or ssDNARNA. In addition, the RAD52 NTD is involved in various DNA repair processes, such as break-induced replication and RNA-templated DNA repair, that are distinct from homologous recombination mediated by the RAD51-interacting region in its CTD. We demonstrated that RAD52 participates in the ALT pathway mediated by MiDAS through its NTD. We propose that the RAD52 NTD is involved in annealing the resected single-stranded telomeres to potential templates (chromosomal telomeres, ECTRs, and TERRA), ultimately leading to elongation processes through break-induced replication, rolling circle amplification, and RNA-templated DNA repair.

A recent study from the telomerase mutant in S. cerevisiae indicated that telomere erosion leads to SUMOylation at telomeres followed by SUMO targeted ubiquitination through E3 ligase slx5/8, and this is crucial for the yeast type II ALT pathway (Churikov et al. 2016). In parallel, human ALT cancer cells exhibit severe DNA replication stresses at telomeres, which leads to telomere clustering events mediated by the SMC5/6 SUMO-ligase complex (Potts and Yu 2007; O'Sullivan et al. 2014; 


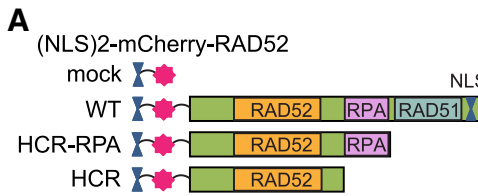

B

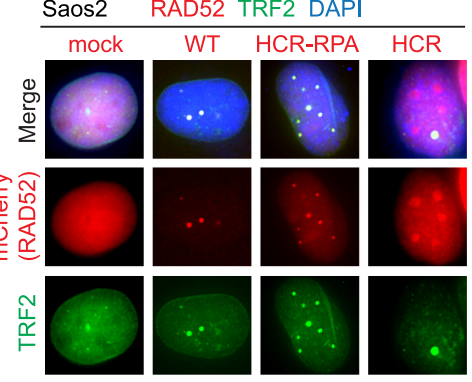

C

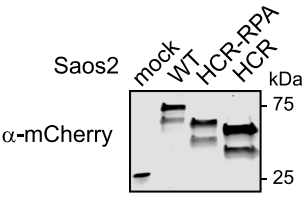

D

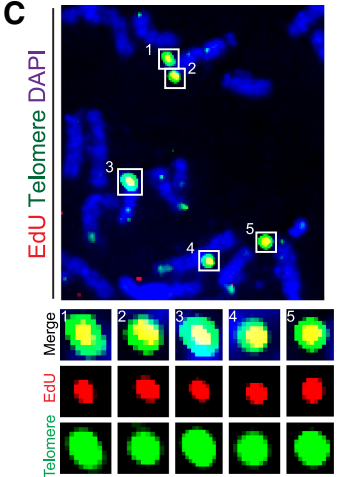

E
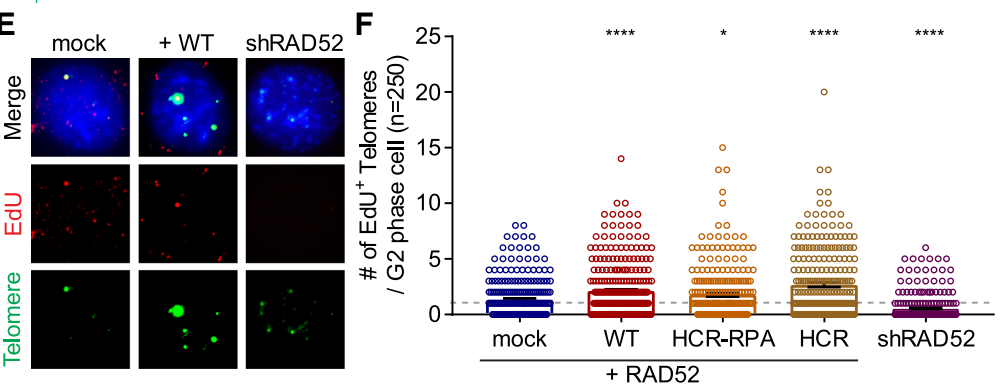

Figure 7. RAD52 facilitates the ALT pathway though its NTD. (A, top) Illustration of human RAD52 proteins fused to NLS and mCherry protein. (Bottom) Immunoblot of Saos2 cells overexpressing RAD52 proteins fused to NLS and mCherry protein. (B) Representative images showing the telomere localization of RAD52 WT and RAD52 mutants. $(C, D)$ Telomeric MiDAS analysis of Saos2 cells in the presence of RAD52 WT or RAD52 mutant overexpression or RAD52 knockdown. $(C)$ Representative images showing MiDAS at telomeres on metaphase spreads. (D) Quantification of telomeric MiDAS as the number of EdU-positive telomeres per metaphase spread. Means \pm SEM. $(E, F)$ G2-phase telomere synthesis analysis of Saos 2 cells in the presence of RAD52 WT or RAD52 mutant overexpression or RAD52 knockdown. (E) Representative images showing G2-phase telomere synthesis at telomeres on metaphase spreads. $(F)$ Quantification of G2-phase telomere synthesis as the number of EdUpositive telomeres per cell. Means \pm SEM. $\left(^{* *}\right) P<$ $\left.0.01 ;{ }^{* * * *}\right) P<0.0001$, unpaired Student's $t$-test.
Osterwald et al. 2015; Cox et al. 2016; Min et al. 2017b). Since most ALT cells do not have an intact G2/M checkpoint (Lovejoy et al. 2012), a subset of ALT cells shows persistent DNA damage responses at telomeres and telomere clustering during mitosis, represented as APB-like foci in metaphase spreads (Cesare et al. 2009), and these ultimately lead to MiDAS at telomeres (Min et al. 2017b). Since APBs are the major sites for telomeric synthesis in ALT cancers, the control of APB disassembly processes before/during/after mitosis could be critical for the ALT pathway as well as cell cycle progression. The APB disassembly processes may be mediated by SUMO targeted ubiquitination, as shown in PML bodies (Geoffroy et al. 2010; Rojas-Fernandez et al. 2014). Testing whether RNF4, the human homolog of slx5/8, is involved in controlling these processes through its SUMO targeted ubiquitin ligase activity may provide additional insights into the human ALT pathway and lead to potential therapeutic approaches in treating ALT tumors.

\section{Materials and methods}

Cell culture and transfection

Cells were cultured at $37^{\circ} \mathrm{C}$ in $5 \% \mathrm{CO}_{2}$ in medium-X $/ 4: 1$ ratio of DMEM and M199) with 10\% cosmic calf serum (Hyclone). When
293FT cells reached $90 \%$ confluency in six-well plates, $10 \mu \mathrm{g}$ of plasmid DNAs and $10 \mu \mathrm{L}$ of Lipofectamine 2000 (Invitrogen) were used for transfection. Cells were analyzed $72 \mathrm{~h}$ after transfection. Cells transfected with telomere clustering scaffolds were harvested by shakeoff, since the transfected cells mostly formed round shapes from G2/M arrest.

\section{Antibodies}

The following antibodies were purchased from the indicated commercial sources: anti-BLM (Santa Cruz Biotechnology, B-4), antimCherry (Novus, [1C51] NBP1-966752), anti-PML (Santa Cruz Biotechnology, PG-M3), anti-RAD52 (Santa Cruz Biotechnology, F-7), anti-RAD51 (Santa Cruz Biotechnology, H-92), anti-RPA (Bethyl, A300-244A), anti-phopho-RPA (S4/S8) (Bethyl, A300245A), and anti-TRF2 (Novus, NB110-57130)

\section{Lentivirus production and infection}

cDNAs (BLM, TRF2, and RAD52 WT and mutants) were inserted into pLenti6/V5_GW/lacZ vector (Invitrogen). The shRNAs targeting RAD52 (shRAD52: V3_LHS_376617) (Wang et al. 2018) and TRF2 (shTRF2_G: TRCN0000018358) (Cesare et al. 2013) were purchased (Open Biosystems). Those lentiviral vectors were cotransfected with packaging vectors pMD2.G (Addgene, 12259) and psPAX2 (Addgene, 12260) in 293FT cells for viral production. Supernatant medium containing produced lentivirus 
were concentrated with homemade $4 \times$ lentivirus concentrator solution (phosphate-buffered saline [PBS] at $\mathrm{pH} 7.2$ containing 1.2 M sodium chloride, 40\% [v/w] PEG-8000).

Generation of RAD52 knockout cells using CRISPR/Cas9 gene targeting

Generation of human RAD52 knockout cells was performed as reported previously (Sotiriou et al. 2016). Briefly, the guide RNA sequence (insert: CACCGTACATAAGTAGCCGCATG GC) targeting exon 3 of the human RAD52 gene was inserted into the px458 vector. For the screening and Sanger sequencing, the CCCTGAGGCAGAGGCTGGGCCCAG and CTCCTACC TTCTGGCCTCCGCC primers were used.

\section{TRF analysis}

The TRF (also known as telomere restriction fragment) was performed as described previously (Lai et al. 2017). Briefly, genomic DNA was purified using Puregene core kit A (Qiagen) following the manufacturer's protocol. DNA samples were digested with HinfI/RsaI (10 U each for $1 \mu \mathrm{g}$ of DNA; New England Biolab). Digested genomic DNAs were run into $0.8 \%$ agarose gel in $1 \times$ trisacetate-ethylenediaminetetraacetic acid (TAE) buffer for $20 \mathrm{~h}$ with $1 \mathrm{~V} / \mathrm{cm}$ followed by depurination with $0.2 \mathrm{M}$ hydrochloride for $15 \mathrm{~min}$, denaturation with $0.5 \mathrm{M}$ sodium hydroxide and $1.5 \mathrm{M}$ sodium chloride for $30 \mathrm{~min}$, and neutralization with $0.5 \mathrm{M}$ Tris$\mathrm{HCl}$ buffer ( $\mathrm{pH} 8.0$ ) and $2 \mathrm{M}$ sodium chloride for $30 \mathrm{~min}$. Gels were transferred using VacuGene (GE Healthcare) and then hybridized with a ${ }^{32} \mathrm{P}$-labeled $\mathrm{C}$ probe to detect the telomere signal. Phospho-images were acquired using a Typhoon FLA 9500 (GE Healthcare) and analyzed using Multi-Gauge version 3.0 software (Fujifilm Life Science).

In-gel hybridization analysis

Digested genomic DNAs were briefly run into a $0.8 \%$ agarose gel in $1 \times$ TAE buffer for $1 \mathrm{~h}$ with $2 \mathrm{~V} / \mathrm{cm}$. The gels were dried at room temperature and hybridized with a ${ }^{32} \mathrm{P}$-labeled $\mathrm{C}$ probe to detect G-rich single-stranded telomere DNA (native gel). Gels were denatured, neutralized, and hybridized with $\mathrm{C}$ probe for total telomere DNA input (denatured gel). The relative ratio of native gel signal to denatured gel signal was calculated.

\section{Immunofluorescence and immuno-FISH}

Cells were fixed in PBS containing 4\% formaldehyde for $5 \mathrm{~min}$. Fixed cells were permeabilized with PBS containing 0.5\% Triton $\mathrm{X}-100$ for $30 \mathrm{~min}$. Cells were incubated with primary antibody in PBS containing $3 \%$ bovine serum albumin (BSA) and $0.1 \%$ Triton $\mathrm{X}-100$ overnight at $4^{\circ} \mathrm{C}$ followed by secondary antibody incubation for $1 \mathrm{~h}$ at room temperature. For immune-FISH, immunestained slides were fixed in PBS containing $4 \%$ formaldehyde for $20 \mathrm{~min}$. Fixed slides were sequentially dehydrated with $70 \%, 90 \%$, and $100 \%$ ethanol for $5 \mathrm{~min}$ each followed by hybridization of a Cy3-TelG probe in hybridization buffer $170 \%$ formamide, $30 \% 2 \times$ saline-sodium citrate $[\mathrm{SSC}], 5 \%$ [v/w] magnesium chloride $\left[\mathrm{MgCl}_{2}\right], 0.0025 \%[\mathrm{v} / \mathrm{w}]$ nucleic acid-blocking reagent [Roche]) overnight. Slides were washed with buffer A (70\% formamide, $30 \% 2 \times$ SSC) for $2 \mathrm{~h}$ and $2 \times$ SSC for $1 \mathrm{~h}$ followed by mounting with VectaShield with DAPI.

\section{Analysis of telomere synthesis during G2 and M phases}

Metaphase spreads and G2-phase cells on slides were hydrated with PBS for 30 min followed by EdU labeling with 6-carboxy- tetramethlyrhodamine fluorescent azide (Invitrogen) in fresh homemade EdU-staining solution (PBS containing $1 \mathrm{mM} \mathrm{CuSO}_{4}$, $2 \mathrm{mM}$ ascorbic acid) for $30 \mathrm{~min}$. Slides were washed vigorously with PBS for $1 \mathrm{~h}$, and then telomere-FISH steps using a FAMTelC probe were followed as described in "Immunofluorescence and Immuno-FISH" above.

\section{Microscope and image acquisition}

Sample images were acquired and analyzed with a DeltaVision deconvolution fluorescence microscope (GE Healthcare). Images were acquired with $100 \times / 1.40$ oil UPLSAPO100X0 1-U2B836 WD $120 \mu \mathrm{m}$ (Olympus). Images were captured with the same intensity and exposure times, and multiple (five) $z$-stack images were taken at $0.2-\mu \mathrm{m}$ intervals. Deconvolution processes were performed using the algorithm with the "conservative" setting, and then the images were projected with the "maximum intensity" method.

\section{Statistical analysis}

Student's two-tailed unpaired $t$-test was used for statistical analysis. The graphs and statistics were generated using Graphpad Prism 7.01 software.

\section{Acknowledgments}

We thank Michael K. Rosen (University of Texas Southwestern Medical Center) and Hongtao Yu (University of Texas Southwestern Medical Center) for sharing reagents and equipment, and Eunhee Choi (University of Texas Southwestern Medical Center) for flow cytometric analysis of the cell cycle and live-cell imaging. We also thank Roger R. Reddel (Children's Medical Research Institute, Australia), Vincent Géli (Research Center of Cancerology of Marseille, France), Jérôme Déjardin (Institute of Human Genetics, France), Dirk Hockemeyer (University of California at Berkeley), and Shang Li (Duke-NUS, Singapore) for helpful discussions. This work was supported by AG01228 from the National Institute on Aging (to W.E.W. and J.W.S.) and the National Cancer Institute (NCI) T32 Cancer Research Training Program (CA124334 to J.M.). We also acknowledge the Harold Simmons NCI Designated Comprehensive Cancer Center Support grant (CA142543) and the Southland Financial Corporation Distinguished Chair in Geriatric Research (J.W.S. and W.E.W.). This work was performed in laboratories constructed with support from National Institutes of Health grant C06 RR30414.

Author contribution: J.M. and J.W.S. conceived the project. J.M. performed the experiments. J.W.S. and W.E.W. supervised the project. J.M. and J.W.S. wrote the manuscript.

\section{References}

Aguzzi A, Altmeyer M. 2016. Phase separation: linking cellular compartmentalization to disease. Trends Cell Biol 26: 547558. doi:10.1016/j.tcb.2016.03.004

Arora R, Lee Y, Wischnewski H, Brun CM, Schwarz T, Azzalin CM. 2014. RNaseH1 regulates TERRA-telomeric DNA hybrids and telomere maintenance in ALT tumour cells. Nat Commun 5: 5220. doi:10.1038/ncomms6220

Banani SF, Rice AM, Peeples WB, Lin Y, Jain S, Parker R, Rosen MK. 2016. Compositional control of phase-separated cellular bodies. Cell 166: 651-663. doi:10.1016/j.cell.2016.06.010

Banani SF, Lee HO, Hyman AA, Rosen MK. 2017. Biomolecular condensates: organizers of cellular biochemistry. Nat Rev Mol Cell Biol 18: 285-298. doi:10.1038/nrm.2017.7 
Barefield C, Karlseder J. 2012. The BLM helicase contributes to telomere maintenance through processing of late-replicating intermediate structures. Nucleic Acids Res 40: 7358-7367. doi:10.1093/nar/gks407

Bernardi R, Pandolfi PP. 2007. Structure, dynamics and functions of promyelocytic leukaemia nuclear bodies. Nat Rev Mol Cell Biol 8: 1006-1016. doi:10.1038/nrm2277

Bhat KP, Cortez D. 2018. RPA and RAD51: fork reversal, fork protection, and genome stability. Nat Struct Mol Biol 25: 446453. doi:10.1038/s41594-018-0075-z

Bhowmick R, Minocherhomji S, Hickson ID. 2016. RAD52 facilitates mitotic DNA synthesis following replication stress. Mol Cell 64: 1117-1126. doi:10.1016/j.molcel.2016.10.037

Bizard AH, Hickson ID. 2014. The dissolution of double Holliday junctions. Cold Spring Harb Perspect Biol 6: a016477. doi:10 $.1101 /$ cshperspect.a016477

Blackford AN, Nieminuszczy J, Schwab RA, Galanty Y, Jackson SP, Niedzwiedz W. 2015. TopBP1 interacts with BLM to maintain genome stability but is dispensable for preventing BLM degradation. Mol Cell 57: 1133-1141. doi:10.1016/j.molcel .2015 .02 .012

Bouchard JJ, Otero JH, Scott DC, Szulc E, Martin EW, Sabri N, Granata D, Marzahn MR, Lindorff-Larsen K, Salvatella X, et al. 2018. Cancer mutations of the tumor suppressor SPOP disrupt the formation of active, phase-separated compartments. Mol Cell 72: 19-36 e18. doi:10.1016/j.molcel.2018.08 .027

Brouwer AK, Schimmel J, Wiegant JC, Vertegaal AC, Tanke HJ, Dirks RW. 2009. Telomeric DNA mediates de novo PML body formation. Mol Biol Cell 20: 4804-4815. doi:10.1091/ mbc.e09-04-0309

Bryan TM, Englezou A, Dalla-Pozza L, Dunham MA, Reddel RR. 1997. Evidence for an alternative mechanism for maintaining telomere length in human tumors and tumor-derived cell lines. Nat Med 3: 1271-1274. doi:10.1038/nm1197-1271

Bugreev DV, Yu X, Egelman EH, Mazin AV. 2007. Novel pro- and anti-recombination activities of the Bloom's syndrome helicase. Genes Dev 21: 3085-3094. doi:10.1101/gad.1609007

Cesare AJ, Reddel RR. 2010. Alternative lengthening of telomeres: models, mechanisms and implications. Nat Rev Genet 11: 319-330. doi:10.1038/nrg2763

Cesare AJ, Kaul Z, Cohen SB, Napier CE, Pickett HA, Neumann AA, Reddel RR. 2009. Spontaneous occurrence of telomeric DNA damage response in the absence of chromosome fusions. Nat Struct Mol Biol 16: 1244-1251. doi:10.1038/nsmb.1725

Cesare AJ, Hayashi MT, Crabbe L, Karlseder J. 2013. The telomere deprotection response is functionally distinct from the genomic DNA damage response. Mol Cell 51: 141-155. doi:10 .1016/j.molcel.2013.06.006

Chen Q, Ijpma A, Greider CW. 2001. Two survivor pathways that allow growth in the absence of telomerase are generated by distinct telomere recombination events. Mol Cell Biol 21: 1819-1827. doi:10.1128/MCB.21.5.1819-1827.2001

Chen Y, Rai R, Zhou ZR, Kanoh J, Ribeyre C, Yang Y, Zheng H, Damay P, Wang F, Tsujii H, et al. 2011. A conserved motif within RAP1 has diversified roles in telomere protection and regulation in different organisms. Nat Struct Mol Biol 18: 213-221. doi:10.1038/nsmb.1974

Cho NW, Dilley RL, Lampson MA, Greenberg RA. 2014. Interchromosomal homology searches drive directional ALT telomere movement and synapsis. Cell 159: 108-121. doi:10 $.1016 /$ j.cell.2014.08.030

Chung I, Leonhardt H, Rippe K. 2011. De novo assembly of a PML nuclear subcompartment occurs through multiple pathways and induces telomere elongation. J Cell Sci 124: 3603-3618. doi: $10.1242 /$ jes. 084681

Chung I, Osterwald S, Deeg KI, Rippe K. 2012. PML body meets telomere: the beginning of an ALTernate ending? Nucleus 3: 263-275. doi:10.4161/nucl.20326

Churikov D, Charifi F, Eckert-Boulet N, Silva S, Simon MN, Lisby M, Géli V. 2016. SUMO-dependent relocalization of eroded telomeres to nuclear pore complexes controls telomere recombination. Cell Rep 15: 1242-1253. doi:10.1016/j.celrep .2016.04.008

Cox KE, Maréchal A, Flynn RL. 2016. SMARCAL1 resolves replication stress at ALT telomeres. Cell Rep 14: 1032-1040. doi:10 .1016/j.celrep.2016.01.011

Déjardin J, Kingston RE. 2009. Purification of proteins associated with specific genomic loci. Cell 136: 175-186. doi:10.1016/j .cell.2008.11.045

Dilley RL, Verma P, Cho NW, Winters HD, Wondisford AR, Greenberg RA. 2016. Break-induced telomere synthesis underlies alternative telomere maintenance. Nature 539: 54 58. doi:10.1038/nature20099

Ditlev JA, Case LB, Rosen MK. 2018. Who's in and who's outcompositional control of biomolecular condensates. I Mol Biol 430: 4666-4684. doi:10.1016/j.jmb.2018.08.003

Doksani Y, de Lange T. 2016. Telomere-internal double-strand breaks are repaired by homologous recombination and PARP1/Lig3-dependent end-joining. Cell Rep 17: 1646-1656. doi:10.1016/j.celrep.2016.10.008

Eladad S, Ye TZ, Hu P, Leversha M, Beresten S, Matunis MJ, Ellis NA. 2005. Intra-nuclear trafficking of the BLM helicase to DNA damage-induced foci is regulated by SUMO modification. Hum Mol Genet 14: 1351-1365. doi:10.1093/hmg/ ddi145

Erdel F, Rippe K. 2018. Formation of chromatin subcompartments by phase separation. Biophys J 114: 2262-2270. doi:10 .1016/j.bpj.2018.03.011

Flynn RL, Cox KE, Jeitany M, Wakimoto H, Bryll AR, Ganem NJ, Bersani F, Pineda JR, Suva ML, Benes CH, et al. 2015. Alternative lengthening of telomeres renders cancer cells hypersensitive to ATR inhibitors. Science 347: 273-277. doi:10.1126/ science. 1257216

Geoffroy MC, Jaffray EG, Walker KJ, Hay RT. 2010. Arsenic-induced SUMO-dependent recruitment of RNF4 into PML nuclear bodies. Mol Biol Cell 21: 4227-4239. doi:10.1091/mbc .e10-05-0449

Hanamshet K, Mazina OM, Mazin AV. 2016. Reappearance from obscurity: mammalian Rad52 in homologous recombination. Genes (Basel) 7: E63. doi:10.3390/genes7090063

Heaphy CM, de Wilde RF, Jiao Y, Klein AP, Edil BH, Shi C, Bettegowda C, Rodriguez FJ, Eberhart CG, Hebbar S, et al. 2011a. Altered telomeres in tumors with ATRX and DAXX mutations. Science 333: 425. doi:10.1126/science.1207313

Heaphy CM, Subhawong AP, Hong SM, Goggins MG, Montgomery EA, Gabrielson E, Netto GJ, Epstein JI, Lotan TL, Westra $\mathrm{WH}$, et al. 2011b. Prevalence of the alternative lengthening of telomeres telomere maintenance mechanism in human cancer subtypes. Am J Pathol 179: 1608-1615. doi:10.1016/j .ajpath.2011.06.018

Hendriks IA, Lyon D, Young C, Jensen LJ, Vertegaal AC, Nielsen ML. 2017. Site-specific mapping of the human SUMO proteome reveals co-modification with phosphorylation. Nat Struct Mol Biol 24: 325-336. doi:10.1038/nsmb.3366

Henson JD, Cao Y, Huschtscha LI, Chang AC, Au AY, Pickett HA, Reddel RR. 2009. DNA C-circles are specific and quantifiable markers of alternative-lengthening-of-telomeres activity. Nat Biotechnol 27: 1181-1185. doi:10.1038/nbt.1587 
Kim NW, Piatyszek MA, Prowse KR, Harley CB, West MD, Ho PL, Coviello GM, Wright WE, Weinrich SL, Shay JW. 1994. Specific association of human telomerase activity with immortal cells and cancer. Science 266: 2011-2015. doi:10 $.1126 /$ science. 7605428

Komosa M, Root H, Meyn MS. 2015. Visualization and quantitative analysis of extrachromosomal telomere-repeat DNA in individual human cells by Halo-FISH. Nucleic Acids Res 43: 2152-2163. doi:10.1093/nar/gkv091

Lai TP, Zhang N, Noh J, Mender I, Tedone E, Huang E, Wright WE, Danuser G, Shay JW. 2017. A method for measuring the distribution of the shortest telomeres in cells and tissues. Nat Commun 8: 1356. doi:10.1038/s41467-017-01291-z

Li B, Oestreich S, de Lange T. 2000. Identification of human Rap1: implications for telomere evolution. Cell 101: 471-483. doi:10 .1016/S0092-8674(00)80858-2

Li F, Kim H, Ji Z, Zhang T, Chen B, Ge Y, Hu Y, Feng X, Han X, Xu $\mathrm{H}$, et al. 2018. The BUB3-BUB1 complex promotes telomere DNA replication. Mol Cell 70: 395-407 e394. doi:10.1016/j .molcel.2018.03.032

Lillard-Wetherell K, Combs KA, Groden J. 2005. BLM helicase complements disrupted type II telomere lengthening in telomerase-negative sgs1 yeast. Cancer Res 65: 5520-5522. doi:10.1158/0008-5472.CAN-05-0632

Lok BH, Carley AC, Tchang B, Powell SN. 2013. RAD52 inactivation is synthetically lethal with deficiencies in BRCA1 and PALB2 in addition to BRCA2 through RAD51-mediated homologous recombination. Oncogene 32: 3552-3558. doi:10 $.1038 /$ onc. 2012.391

Lovejoy CA, Li W, Reisenweber S, Thongthip S, Bruno J, de Lange T, De S, Petrini JH, Sung PA, Jasin M, et al. 2012. Loss of ATRX, genome instability, and an altered DNA damage response are hallmarks of the alternative lengthening of telomeres pathway. PLoS Genet 8: e1002772. doi:10.1371/ journal.pgen.1002772

Lundblad V, Blackburn EH. 1993. An alternative pathway for yeast telomere maintenance rescues est1- senescence. Cell 73: 347-360. doi:10.1016/0092-8674|93/90234-H

Lundblad V, Szostak JW. 1989. A mutant with a defect in telomere elongation leads to senescence in yeast. Cell 57: 633643. doi:10.1016/0092-8674(89)|90132-3

Lydeard JR, Jain S, Yamaguchi M, Haber JE. 2007. Break-induced replication and telomerase-independent telomere maintenance require Pol32. Nature 448: 820-823. doi:10.1038/ nature 06047

Mao P, Liu J, Zhang Z, Zhang H, Liu H, Gao S, Rong YS, Zhao Y. 2016. Homologous recombination-dependent repair of telomeric DSBs in proliferating human cells. Nat Commun 7: 12154. doi: $10.1038 /$ ncomms 12154

Marzec P, Armenise C, Pérot G, Roumelioti FM, Basyuk E, Gagos S, Chibon F, Déjardin J. 2015. Nuclear-receptor-mediated telomere insertion leads to genome instability in ALT cancers. Cell 160: 913-927. doi:10.1016/j.cell.2015.01.044

Mazina OM, Keskin H, Hanamshet K, Storici F, Mazin AV. 2017. Rad52 inverse strand exchange drives RNA-templated DNA double-strand break repair. Mol Cell 67: 19-29 e13. doi:10 .1016/j.molcel.2017.05.019

McDevitt S, Rusanov T, Kent T, Chandramouly G, Pomerantz RT. 2018. How RNA transcripts coordinate DNA recombination and repair. Nat Commun 9: 1091. doi:10.1038/s41467018-03483-7

Min J, Wright WE, Shay JW. 2017a. Alternative lengthening of telomeres can be maintained by preferential elongation of lagging strands. Nucleic Acids Res 45: 2615-2628. doi:10.1093/ nar/gkw1295
Min J, Wright WE, Shay JW. 2017b. Alternative lengthening of telomeres mediated by mitotic DNA synthesis engages break-induced replication processes. Mol Cell Biol 37: e00226-17. doi:10.1128/MCB.00226-17

Minocherhomji S, Ying S, Bjerregaard VA, Bursomanno S, Aleliunaite A, Wu W, Mankouri HW, Shen H, Liu Y, Hickson ID. 2015. Replication stress activates DNA repair synthesis in mitosis. Nature 528: 286-290. doi:10.1038/nature16139

Muntoni A, Neumann AA, Hills M, Reddel RR. 2009. Telomere elongation involves intra-molecular DNA replication in cells utilizing alternative lengthening of telomeres. Hum Mol Genet 18: $1017-1027$. doi:10.1093/hmg/ddn436

Nabetani A, Ishikawa F. 2009. Unusual telomeric DNAs in human telomerase-negative immortalized cells. Mol Cell Biol 29: 703-713. doi:10.1128/MCB.00603-08

Nimonkar AV, Genschel J, Kinoshita E, Polaczek P, Campbell JL, Wyman C, Modrich P, Kowalczykowski SC. 2011. BLMDNA2-RPA-MRN and EXO1-BLM-RPA-MRN constitute two DNA end resection machineries for human DNA break repair. Genes Dev 25: 350-362. doi:10.1101/gad.2003811

Oganesian L, Karlseder J. 2011. Mammalian 5' C-rich telomeric overhangs are a mark of recombination-dependent telomere maintenance. Mol Cell 42: 224-236. doi:10.1016/j.molcel .2011.03.015

Osterwald S, Deeg KI, Chung I, Parisotto D, Worz S, Rohr K, Erfle H, Rippe K. 2015. PML induces compaction, TRF2 depletion and DNA damage signaling at telomeres and promotes their alternative lengthening. I Cell Sci 128: 1887-1900. doi:10 $.1242 /$ jcs. 148296

O'Sullivan RJ, Arnoult N, Lackner DH, Oganesian L, Haggblom C, Corpet A, Almouzni G, Karlseder J. 2014. Rapid induction of alternative lengthening of telomeres by depletion of the histone chaperone ASF1. Nat Struct Mol Biol 21: 167-174. doi:10 $.1038 / \mathrm{nsmb} .2754$

Özer Ö, Bhowmick R, Liu Y, Hickson ID. 2018. Human cancer cells utilize mitotic DNA synthesis to resist replication stress at telomeres regardless of their telomere maintenance mechanism. Oncotarget 9: 15836-15846. doi:10.18632/oncotarget .24745

Pan X, Drosopoulos WC, Sethi L, Madireddy A, Schildkraut CL, Zhang D. 2017. FANCM, BRCA1, and BLM cooperatively resolve the replication stress at the ALT telomeres. Proc Nat1 Acad Sci 114: E5940-E5949. doi:10.1073/pnas.1708065114

Potts PR, Yu H. 2007. The SMC5/6 complex maintains telomere length in ALT cancer cells through SUMOylation of telomerebinding proteins. Nat Struct Mol Biol 14: 581-590. doi:10 $.1038 / \mathrm{nsmb} 1259$

Ramamoorthy M, Smith S. 2015. Loss of ATRX suppresses resolution of telomere cohesion to control recombination in ALT cancer cells. Cancer Cell 28: 357-369. doi:10.1016/j.ccell .2015.08.003

Rojas-Fernandez A, Plechanovová A, Hattersley N, Jaffray E, Tatham MH, Hay RT. 2014. SUMO chain-induced dimerization activates RNF4. Mol Cell 53: 880-892. doi:10.1016/j .molcel.2014.02.031

Root H, Larsen A, Komosa M, Al-Azri F, Li R, Bazett-Jones DP, Stephen Meyn M. 2016. FANCD2 limits BLM-dependent telomere instability in the alternative lengthening of telomeres pathway. Hum Mol Genet 25: 3255-3268. doi:10.1093/hmg/ ddw 175

Sfeir A, Kosiyatrakul ST, Hockemeyer D, MacRae SL, Karlseder J, Schildkraut CL, de Lange T. 2009. Mammalian telomeres resemble fragile sites and require TRF1 for efficient replication. Cell 138: 90-103. doi:10.1016/j.cell.2009.06.021 
Singleton MR, Wentzell LM, Liu Y, West SC, Wigley DB. 2002. Structure of the single-strand annealing domain of human RAD52 protein. Proc Nat1 Acad Sci 99: 13492-13497. doi:10 .1073/pnas.212449899

Sobinoff AP, Pickett HA. 2017. Alternative lengthening of telomeres: DNA repair pathways converge. Trends Genet 33: 921-932. doi:10.1016/j.tig.2017.09.003

Sobinoff AP, Allen JA, Neumann AA, Yang SF, Walsh ME, Henson JD, Reddel RR, Pickett HA. 2017. BLM and SLX4 play opposing roles in recombination-dependent replication at human telomeres. EMBO I 36: 2907-2919. doi:10.15252/ embj.201796889

Sotiriou SK, Kamileri I, Lugli N, Evangelou K, Da-Ré C, Huber F, Padayachy L, Tardy S, Nicati NL, Barriot S, et al. 2016. Mammalian RAD52 functions in break-induced replication repair of collapsed DNA replication forks. Mol Cell 64: 1127-1134. doi:10.1016/j.molcel.2016.10.038

Stavropoulos DJ, Bradshaw PS, Li X, Pasic I, Truong K, Ikura M, Ungrin M, Meyn MS. 2002. The Bloom syndrome helicase BLM interacts with TRF2 in ALT cells and promotes telomeric DNA synthesis. Hum Mol Genet 11: 3135-3144. doi:10.1093/hmg/11.25.3135

Teng SC, Zakian VA. 1999. Telomere-telomere recombination is an efficient bypass pathway for telomere maintenance in Saccharomyces cerevisiae. Mol Cell Biol 19: 8083-8093. doi:10 $.1128 /$ MCB.19.12.8083

Teng SC, Chang J, McCowan B, Zakian VA. 2000. Telomeraseindependent lengthening of yeast telomeres occurs by an abrupt Rad50p-dependent, Rif-inhibited recombinational process. Mol Cell 6: 947-952. doi:10.1016/S1097-2765(05) 00094-8

Teng Y, Yadav T, Duan M, Tan J, Xiang Y, Gao B, Xu J, Liang Z, Liu Y, Nakajima S, et al. 2018. ROS-induced R loops trigger a transcription-coupled but BRCA1/2-independent homologous recombination pathway through CSB. Nat Commun 9: 4115. doi:10.1038/s41467-018-06586-3
Verma P, Greenberg RA. 2016. Noncanonical views of homologydirected DNA repair. Genes Dev 30: 1138-1154. doi:10.1101/ gad.280545.116

Verma P, Dilley RL, Zhang T, Gyparaki MT, Li Y, Greenberg RA. 2019. RAD52 and SLX4 act nonepistatically to ensure telomere stability during alternative telomere lengthening. Genes Dev 33: 221-235. doi:10.1101/gad.319723.118

Wang J, Chen J, Gong Z. 2013. TopBP1 controls BLM protein level to maintain genome stability. Mol Cell 52: 667-678. doi:10 .1016/j.molcel.2013.10.012

Wang H, Li S, Oaks J, Ren J, Li L, Wu X. 2018. The concerted roles of FANCM and Rad52 in the protection of common fragile sites. Nat Commun 9: 2791. doi:10.1038/s41467-018-05066-y

Wheeler RJ, Hyman AA. 2018. Controlling compartmentalization by non-membrane-bound organelles. Philos Trans $R$ Soc Lond B Biol Sci 373. doi:10.1098/rstb.2017.0193

Yankiwski V, Marciniak RA, Guarente L, Neff NF. 2000. Nuclear structure in normal and Bloom syndrome cells. Proc Nat1 Acad Sci 97: 5214-5219. doi:10.1073/pnas.090525897

Yeager TR, Neumann AA, Englezou A, Huschtscha LI, Noble JR, Reddel RR. 1999. Telomerase-negative immortalized human cells contain a novel type of promyelocytic leukemia (PML) body. Cancer Res 59: 4175-4179.

Zhang JM, Yadav T, Ouyang J, Lan L, Zou L. 2019. Alternative lengthening of telomeres through two distinct break-induced replication pathways. Cell Rep 26: P955-P968.e3. doi:10 $.1016 /$ j.celrep.2018.12.102

Zhu J, Zhu S, Guzzo CM, Ellis NA, Sung KS, Choi CY, Matunis MJ. 2008. Small ubiquitin-related modifier (SUMO) binding determines substrate recognition and paralog-selective SUMO modification. I Biol Chem 283: 29405-29415. doi:10 $.1074 /$ jbc.M803632200

Zimmermann M, Kibe T, Kabir S, de Lange T. 2014. TRF1 negotiates TTAGGG repeat-associated replication problems by recruiting the BLM helicase and the TPP1/POT1 repressor of ATR signaling. Genes Dev 28: 2477-2491. doi:10.1101/gad .251611 .114 


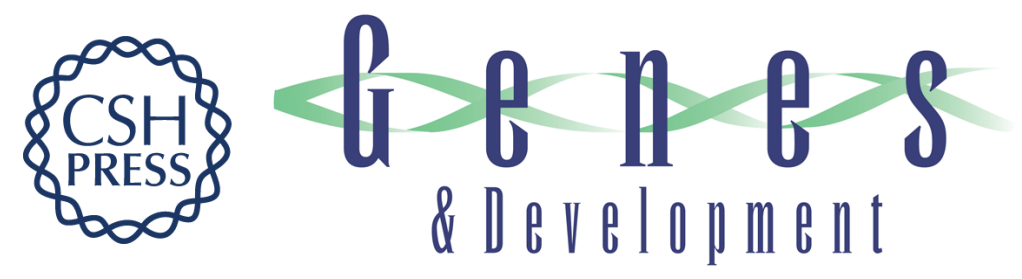

\title{
Clustered telomeres in phase-separated nuclear condensates engage mitotic DNA synthesis through BLM and RAD52
}

\author{
Jaewon Min, Woodring E. Wright and Jerry W. Shay \\ Genes Dev. 2019, 33: originally published online June 6, 2019 \\ Access the most recent version at doi:10.1101/gad.324905.119
}

\section{Supplemental http://genesdev.cshlp.org/content/suppl/2019/06/04/gad.324905.119.DC1 Material}

References This article cites 86 articles, 23 of which can be accessed free at: http://genesdev.cshlp.org/content/33/13-14/814.full.html\#ref-list-1

Creative This article is distributed exclusively by Cold Spring Harbor Laboratory Press for the first Commons six months after the full-issue publication date (see

License http://genesdev.cshlp.org/site/misc/terms.xhtml). After six months, it is available under a Creative Commons License (Attribution-NonCommercial 4.0 International), as described at http://creativecommons.org/licenses/by-nc/4.0/.

Email Alerting Receive free email alerts when new articles cite this article - sign up in the box at the top Service right corner of the article or click here.

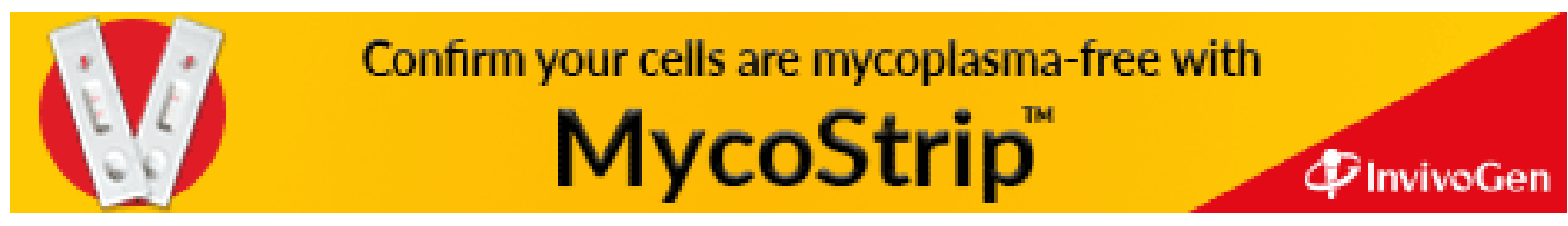

